Revue française de pédagogie

凹PÉDAGOGIE

\author{
Recherches en éducation
}

191 | avril-mai-juin 2015

Les descendants d'immigrés à l'école

\title{
BARON Georges-Louis, BRUILLARD Éric \& DROT- DELANGE Béatrice (dir.). Informatique en éducation : perspectives curriculaires et didactiques
}

Clermont-Ferrand : Presses universitaires Blaise-Pascal, 2015, 316 p.

\section{Cédric Fluckiger}

\section{Q OpenEdition}

\section{Journals}

Édition électronique

URL : http://journals.openedition.org/rfp/4778

DOI : $10.4000 /$ rfp. 4778

ISSN : $2105-2913$

Éditeur

ENS Éditions

Édition imprimée

Date de publication : 30 juin 2015

Pagination : 137-138

ISBN : 978-2-84788-772-3

ISSN : 0556-7807

\section{Référence électronique}

Cédric Fluckiger, «BARON Georges-Louis, BRUILLARD Éric \& DROT-DELANGE Béatrice (dir.)

Informatique en éducation : perspectives curriculaires et didactiques", Revue française de pédagogie [En

ligne], 191 | avril-mai-juin 2015, mis en ligne le 30 juin 2015, consulté le 23 septembre 2020. URL

http://journals.openedition.org/rfp/4778 ; DOI : https://doi.org/10.4000/rfp.4778 


\section{Notes critiques}

BARON Georges-Louis, BRUILLARD Éric \& DROT-DELANGE Béatrice (dir.). Informatique en éducation : perspectives curriculaires et didactiques. Clermont-Ferrand : Presses universitaires Blaise-Pascal, 2015, 316 p.

"L'informatique» ou "le numérique» sont omniprésents dans le champ éducatif, fût-ce sous la forme de dénominations génériques dont personne ne sait précisément à quoi elles renvoient. En témoignent les prises de position politiques sur "l'école numérique», sur la «littératie numérique, indispensables à la formation du futur citoyen » (Najat Vallaud-Belkacem) ou les divers rapports parlementaires ou institutionnels parus ces derniers mois. Dans les nouveaux projets de programme des cycles 2-3-4 (tels qu'ils ont été rendus publics à l'heure où ces lignes sont écrites), le terme «informatique » apparaît 31 fois, le terme «numérique» plus de 200 fois (sans compter le mot "numérique» employé dans son sens mathématique). Près de vingt ans après la disparition de l'option Informatique des lycées, la nouvelle option Informatique et sciences du numérique est en voie de généralisation.

Cette présence du numérique dans les discours pourrait faire penser que des recherches en sciences de l'éducation, et notamment en didactique, seraient également importantes et bien installées. II n'en est cependant rien. Comme le rappelle l'introduction de l'ouvrage, si la didactique de l'informatique a connu dans les années 1970-1980 une certaine importance, reflétant les préoccupations liées à la programmation et aux vertus éducatives que lui voyaient les pionniers de l'informatique scolaire, le dernier colloque de didactique de l'informatique a eu lieu... en 1996.

Certes, I'informatique n'est pas une matière scolaire (avec des horaires, des programmes, un corps d'enseignants, etc.). Certes, les auteurs rappellent que les préoccupations depuis vingt ans ont davantage porté sur l'instrumentation pédagogique que sur les contenus informatiques. Pourtant, des contenus indéniablement informatiques existent bel et bien, pris en charge par d'autres matières (des tableurs-grapheurs en mathématiques à la technologie, en passant par des savoirs documentaires). À l'école, on apprend à désigner les composants d'un ordinateur, à envoyer un mail, à connaître les rudiments du droit à l'image, etc.

C'est bien à ces contenus qu'est consacré cet ouvrage, et non à l'idée d'un "hypothétique "outil informatique" ne nécessitant pas de formation » (p. 12), idée qui s'est répandue dans les discours politiques et institutionnels, sensibles à une sorte d'injonction de modernité, quand ce n'est pas aux discours marchands.

L'intérêt de l'ouvrage est précisément de mettre en avant certains de ces contenus ainsi que les travaux qui les constituent en objets de recherche.

L'ouvrage regroupe des contributions issues du colloque Dida\&STIC, qui s'est tenu à Clermont-Ferrand en octobre 2013. Le nom même du colloque illustre l'évolution des préoccupations des chercheurs, en lien avec certaines évolutions de la place de l'informatique à l'école. En effet, les colloques Didapro pour «Didactique des progiciels» (c'est-à-dire des produits logiciels), qui se sont tenus depuis 2006, ont pris une appellation nouvelle, «Dida\&STIC», afin de rendre visible la variété des approches possibles de l'informatique et du numérique en éducation.

Les contributions à l'ouvrage s'organisent en trois parties. La première adopte une perspective internationale, questionnant la place de l'informatique dans les curricula de différents pays ou région: Israël (J. Gal-Ezer), Bavière (P. Hubwieser), Belgique (J. Henry \& N. Joris) et 
France (G.-L. Baron, B. Drot-Delange, M. Grandbastien \& F. Tort). La diversité des situations permet de mesurer qu'il n'y a pas de fatalité à ce que «les décideurs [considèrent] que l'informatique est déjà présente à l'école via l'ordinateur» (p. 13).

La deuxième partie constitue un prolongement des colloques Didapro, en se focalisant sur les usages informatiques dans d'autres disciplines scolaires ou au service d'autres apprentissages. Sont successivement examinés le cas des recherches d'information sur le Web (C. Ladage), de l'enseignement du design (É. Tortochot), la certification des compétences numériques (E. Vandeput) et l'enseignement du modèle objet en Bavière (P. Hubwieser). II ressort de l'analyse de ces usages et enseignements la «nécessité d'une compréhension conceptuelle, qui réfère à l'informatique» (p.15).

La dernière partie se centre sur l'enseignement de contenus directement et explicitement référés à la science informatique ou à des domaines proches, comme la robotique : de la programmation à l'école maternelle (V. Komis \& A. Misirli) à l'initiation à la programmation d'étudiants de master (G.-L. Baron \&E. Voulgre), en passant par la visualisation dynamique de programmes (M. Gautier \& B. Wrobel-Dautcourt) ou la programmation de robots (C. Nijimbere, L. Boulc'h \& G.-L. Baron). La partie se conclut par un chapitre rétrospectif de J. Rogalski, revenant sur les liens entre la psychologie de la programmation et la didactique de l'informatique.

Les contributions rassemblées dans cet ouvrage donnent à voir, à travers la variété des approches didactiques de l'informatique en éducation, une voie de résolution possible de la tension (qui sous-tend les discours institutionnels depuis plus de vingt ans) entre l'informatique outil d'enseignement/apprentissage et l'informatique objet d'enseignement, puisque les outils eux-mêmes demandent un apprentissage et une forme de compréhension des mécanismes sousjacents en jeu.

II semble qu'il s'agit là d'une étape dans la structuration d'une communauté de recherche intéressée aux apprentissages spécifiques de l'informatique et du numérique. Reste que si la structuration d'une didactique de l'informatique ne gagnerait évidemment rien à une réduction théorique autour d'une théorie unique, l'ouvrage permet aussi de mesurer que ce champ peine encore à faire communauté autour de ce qu'il a de spécifique : la centration sur les phénomènes d'enseignement/apprentissage des contenus informa- des approches d'autres disciplines que des autres didactiques. Or, dans la plupart des chapitres, les références croisées sont rares et empruntent plus fréquemment à d'autres disciplines, sociologie ou psychologie, qu'à la didactique.

Cette richesse, cette ouverture aux disciplines proches et le refus d'un enfermement autour de questionnements ou d'un cadre théorique unique constituent une réelle opportunité pour qu'émerge un corps de questions et de concepts partagés, pertinents pour les contenus et les usages informatiques, qui pourront en retour nourrir les disciplines voisines, en premier lieu les autres didactiques, disciplinaires ou comparées.

Cédric Fluckiger Université Lille 3, Cirel

BECQUEMIN Michèle \& MONTANDON Christiane (dir.). Les institutions à l'épreuve des dispositifs. Les recompositions de l'éducation et de l'intervention sociale. Rennes : Presses universitaires de Rennes, 2014, 232 p.

"Comment comprendre les rapports complexes et souvent tendus entre les institutions et les dispositifs dans les champs de l'éducation et de l'intervention sociale?» Le livre coordonné par Michèle Becquemin et Christiane Montandon part d'un double constat : notre modernité avancée se méfie des cadres institutionnels qu'elle juge arbitraires; cette méfiance s'incarne dans la profusion de dispositifs de circonstance, censés répondre plus habilement qu'une lourde bureaucratie aux besoins politiques. Empilement, enchevêtrement, et finalement envahissement des métiers de l'humain par ces dispositifs foisonnants : le phénomène observé serait à la fois préoccupant et difficile à contenir, car partie intégrante d'une puissante remise en cause de l'État-providence. "Redéploiement », «réajustement», « renouvellement», voire «phagocytage» de l'ordre institutionnel par une logique auxiliaire ou carrément concurrente de dispositifs fonctionnels : c'est en somme cette hypothèse que les auteurs ${ }^{1}$ souhaitent mettre en discussion.

1 Le masculin utilisé dans ce texte est purement grammatical. II renvoie à des collectifs composés aussi bien d'hommes que de 